\title{
Acoustic Parameters for Optimizing Lung Volume During High- Frequency Oscillatory Ventilation in Infants with Respiratory Distress Syndrome: A Feasibility Study
}

\author{
Oded Luria ${ }^{1}$, David Kohelet ${ }^{2}$ and Ofer Barnea ${ }^{1, *}$ \\ ${ }^{I}$ Department of Biomedical Engineering, Tel Aviv University, Israel \\ ${ }^{2}$ Department of Neonatology, Wolfson Medical Center, Holon, Israel and the Sackler School of Medicine, Tel Aviv Uni- \\ versity, Israel
}

\begin{abstract}
During high-frequency oscillatory ventilation (HFOV), the primary variable affecting lung volume is the mean airway pressure (MAP). To effectively maintain lung recruitment and optimal gas exchange without overstretching (or collapsing) the lung, MAP should be set between the lower and upper inflection points of the pressure-volume curve of the lung. At present, there is no efficacious means that allows the neonatologist to determine the MAP (optimal MAP) which attains optimal lung expansion and avoids overdistension. Thus, MAP is usually adjusted by trial and error or by clinical experience of the user. In this study, we investigated the acoustic properties of the neonate lung in six newborns undergoing high frequency oscillatory ventilation, to assess its usefulness as a means for determining optimal mean airway pressure. We found that the shape of the acoustic reflection-pressure curve was similar to the shape of the known pressure-volume curve. In all subjects, the estimated range of MAP was in congruence with the pressure chosen by the neonatologist. The acoustic measurements indicated of an increase in lung volume following administration of exogenous surfactant. Hysteresis in the amplitude of acoustic reflection was measured as expected. Our results indicate that the acoustic technique provides useful information about the state of lung recruitment during HFOV and may be helpful in identifying the adequate MAP for optimal lung expansion without overdistension.
\end{abstract}

Keywords: High frequency oscillatory ventilation, lung-protective ventilation strategies, mean airway pressure, neonatology, respiratory distress syndrome, lung hysteresis, preterm infant.

\section{BACKGROUND}

Mechanical ventilation can induce lung injury, particularly in premature and diseased lungs. There is increasing evidence that high peak inspiratory pressures and repetitive end-expiratory collapse are major determinants of lung injury [1]. Efforts to minimize ventilator-induced lung injury in infants with respiratory distress syndrome (RDS) have focused on the potential role of High-frequency oscillatory ventilation (HFOV), which has been studied in newborn infants with RDS. HFOV is a method of ventilation in which alveolar gas exchange is maintained by pressure swings initiating small displacements of ventilatory gases, considerably smaller than conventional tidal volumes, at frequencies generally from $5-20 \mathrm{~Hz}[2,3]$. HFOV potentially offers the ideal combination of minimum tidal volume while maintaining maximal recruitment, provided sufficient end-expiratory lung volume is maintained. Since it allows higher endexpiratory pressures with lower peak inspiratory pressures and higher mean airway pressure (MAP), HFOV has become a standard care for the ventilatory management of critically ill neonates $[4,5]$. A critical procedure in HFOV is the adjustment of MAP to a level that allows optimal gas exchange without overstretching (or collapsing) the lung tissue [2,6-9].

*Address correspondence to this author at the Department of Biomedical Engineering, Tel Aviv University, Israel; Tel: +972-3-640-8658; Fax: +9723-640-5830; E-mail: barneao@eng.tau.ac.il
Presently, MAP is adjusted by trial and error or by clinical experience of the user. Increasing the MAP along the pressure volume curve will result in increasing lung volume. When lung volume increases above the critical opening pressure of the lung compliance will improve. Over time, lung compliance continues to improve and when not compensated by decreased MAP, results in increasing lung volume and eventually, overdistension. This may cause compression of the alveolar vascular bed and increase vascular resistance, decreased preload and cardiac output.

The pressure-volume curve is often used to illustrate the balance between overdistension and recruitment. As MAP is increased from functional residual capacity (FRC), an abrupt change in the lung compliance is often evident, particularly in injured or surfactant deficient lungs. The lower inflection point (LIP) of the pressure-volume curve may represent the approximate pressure (volume) at which lung units are recruited. The upper inflection point (UIP) at which lung compliance decreases at higher airway pressure is thought to reflect the point at which alveoli are becoming overdistended, and therefore potentially damaged. The use of MAP in a "safe zone", between LIP and UIP is considered as sufficient to provide adequate oxygenation and to prevent alveolar overdistension $[1,10,11]$. Lung overdistension can be assessed using chest radiography (flattened diaphragm and/or bulging in the intercostals space). However, estimation of lung volume using chest X-Ray [12] was shown to be 


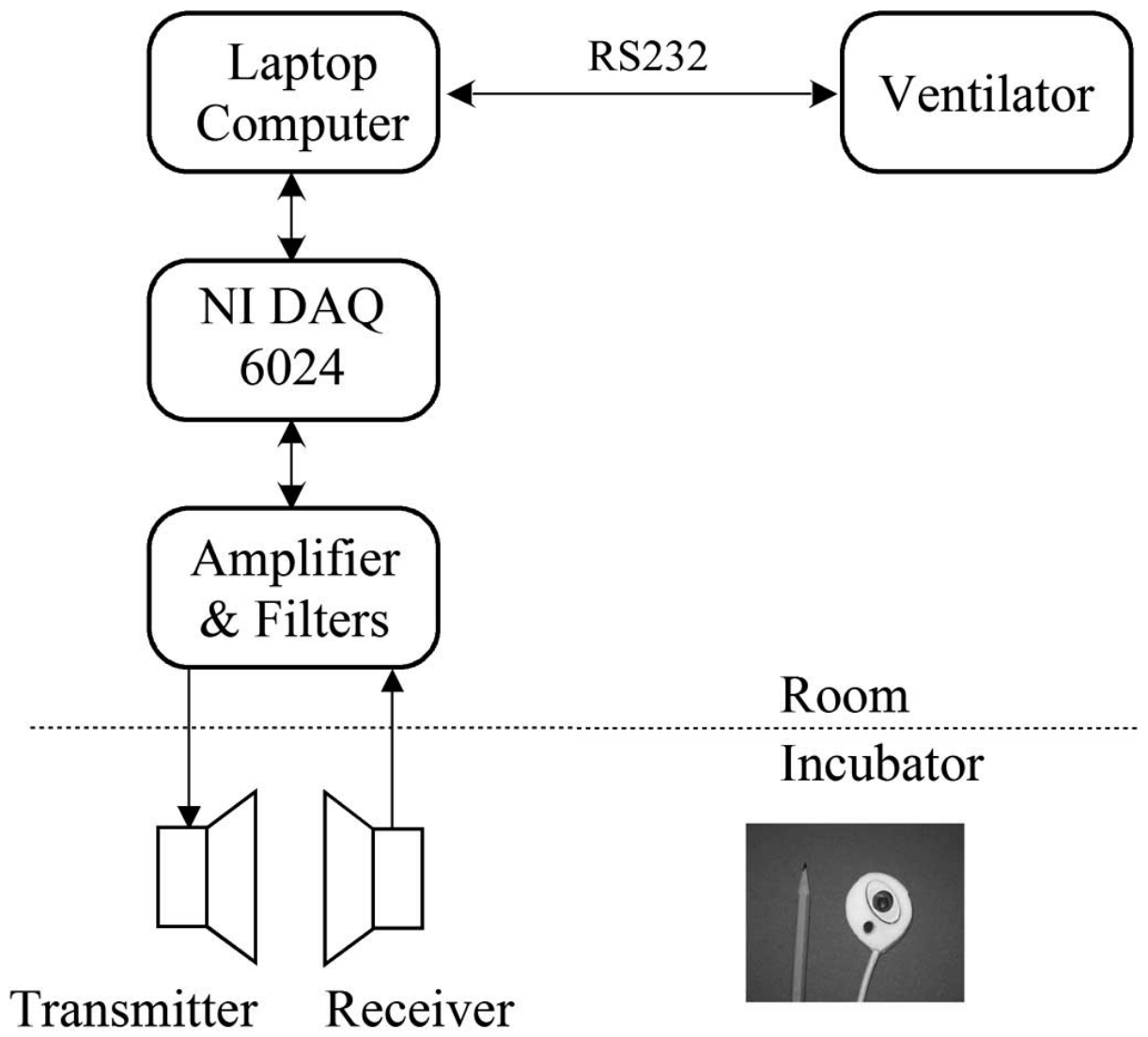

Fig. (1). Schematic diagram of the acoustic measurement system. LPF: low-pass filter; HPF: high-pass filter. Bottom right: the acoustic sensor, comprising of the acoustic transmitter and receiver. A pencil is located at the side for size reference.

inaccurate [13]. Consequently, there has been increasing interest in the role of novel techniques which may describe lung volume during HFOV. Examples are respiratory inductive plethysmography (RIP) $[14,15]$ and electrical impedance tomography (EIT) [16].

To the best of our knowledge, the use of acoustic techniques for the assessment of lung volume in ventilated neonates during HFOV has not been reported yet. In other fields, there have been various attempts to use sound waves in the investigation of lung mechanics. Rice was first to demonstrate that lung volume correlates with sound transmission [17]. His work has been followed by extensive work of other researchers [18-21] who showed that sound attenuation through lung parenchyma has a strong relationship with lung density. Parenchymal sound speed was estimated to be between $23 \mathrm{~m} / \mathrm{s}$ and $70 \mathrm{~m} / \mathrm{s}$ [17], depending on air content. Sound attenuation of the lung parenchyma was found to increase with frequency [18]. In addition, sound transient time through the lungs was found to decrease with increasing lung volume [22]. High-frequency sounds were shown to travel further within the airway-branching structure, while lowfrequency sounds appear to exit predominantly the large airways via wall motion $[19,23]$. In infants (within three days after birth), the acoustic spectrum was found to contain less power below $300 \mathrm{~Hz}$, compared with children and adults [24]. In the isolated lung of near-term fetal sheep, both the velocity of audible sound through the lung and the degree of sound attenuation were correlated to the degree of lung infla- tion, particularly in high lung densities [25]. Attenuation was also shown to be frequency-dependent at changing lung volumes in the adult lung [24]. In acute cases of lung injury and pneumothorax, the changes in the acoustic properties of the lung were found to be correlative to the degree of injury [26, 27]. Also, the acoustic properties of the adult lung were used for lung imaging [28].

We present a feasibility study of an acoustic system that may help to determine an optimal MAP during HFOV. We hypothesized that the acoustic properties of the neonate lung reflect the degree of lung recruitment, and that the degree of acoustic reflection can be used to estimate changes in lung volume change during HFOV. Our results indicate that the technique provides useful information about the state of lung recruitment during HFOV and may indicate on the optimal range of MAP.

\section{MATERIALS AND METHODS}

\subsection{The Acoustic Measurement System}

A block diagram of the experimental setup is shown in Fig. (1). The acoustic measurement system consisted of a computer, A/D card (National Instruments PCI-6024), acoustic sensor consisting of an acoustic transmitter (Horn, $4.5 \times 3.0 \mathrm{~mm}$, sensitivity of $44 \mathrm{~dB} \pm 3 \mathrm{~dB}$ ), an acoustic receiver (254-PR652, Kobitone audio, radii $24 \mathrm{~mm}$, frequency range: $300-4000 \mathrm{~Hz}$ ), and an electronic circuit. The electronic circuit was operated by a stable $+5 \mathrm{~V}$ supply, generated by a USB 

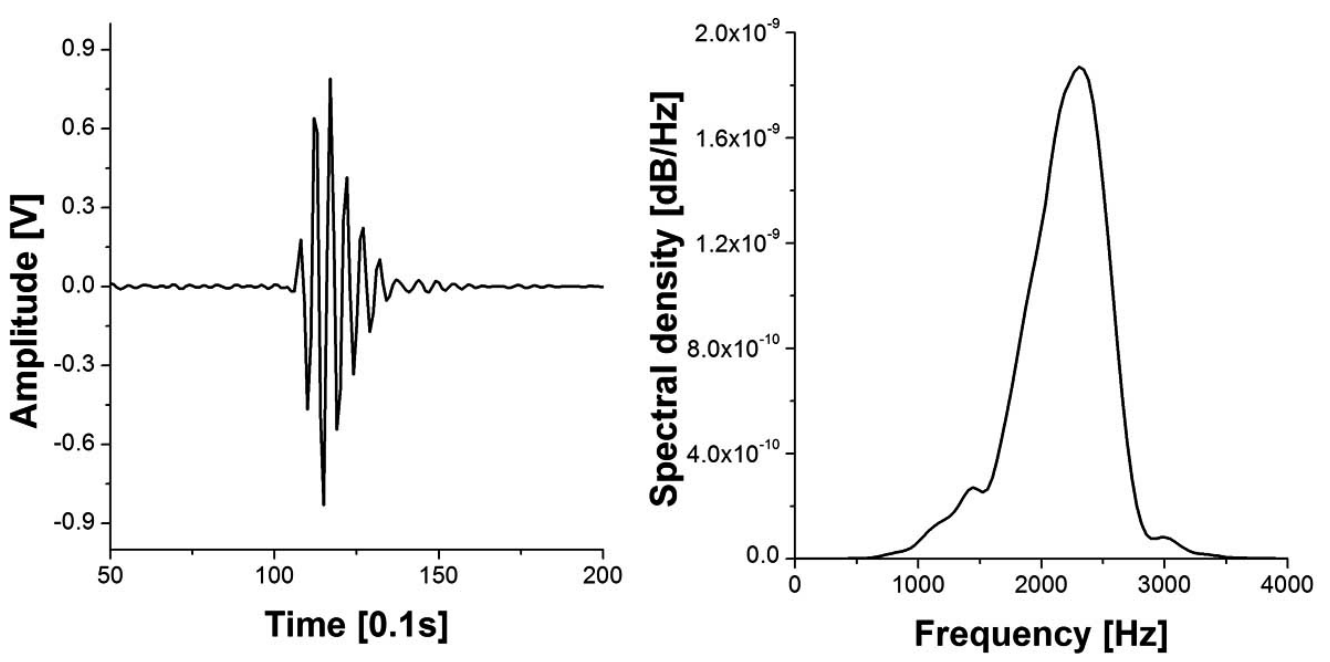

Fig. (2). The transmitted signal in the time (left panel) and frequency domains (right panel).

connection between the computer and the electronic circuit. The computer was connected by a RS232 communication cable to a Stephanie ventilator (Stephan Biomedical Incorporation, Gackenbach, Germany). The electronic circuit and computer were tested with a safety analyzer to ensure it meets the safety standards of Israeli Ministry of Health and Israeli Institute of Standards regarding the usage of medical equipment (IEC601-1).

Acoustic signals (sonic frequency) in the form of a series of bursts (pulse repetition frequency of $25 \mathrm{~Hz}$ ) were generated by the A/D card and transmitted by the acoustic transmitter to the subject chest. Each pulse contained spectral energy in frequencies between 1300-3000 Hz, with peak spectral density at $2500 \mathrm{~Hz}$. Fig. (2) presents the transmitted signal in the time and frequency domains. The acoustic signal reflected by the lungs was recorded by the receiver and filtered by the electronic circuit (bandwidth of 100-3500 $\mathrm{Hz}$ ). The signal was sampled at frequency of $10 \mathrm{KHz}$ and digitized by a National Instrument PCI-6024E card. Ventilatory parameters (i.e., mean airway pressure, ventilation frequency) were transferred from the Stephanie ventilator to the computer using RS232 communication protocol. All data was saved for offline analysis.

\subsection{Clinical Protocol}

The Institutional Review Board for Human Experimentation in the Edith Wolfson Medical Center approved the protocol of this trial and all parents provided written informed consent.

The infants were intubated in the delivery room, and ventilated using intermittent positive pressure ventilation during transport to the neonatal intensive care unit (NICU), where HFOV served as the primary and exclusive mode of ventilation, irrespective of their underlying lung disease. HFOV was performed using a Stephanie ventilators. Oscillatory frequency was kept unchanged at $15 \mathrm{~Hz}$ (900 cycles/minute) throughout the ventilation treatment. The frequency of $15 \mathrm{~Hz}$ was used for reasons of convenience and practicality, since it allows chest vibrations to be used as a clinically detectable index of transmission of pressure oscillations to the lungs. This visual feedback is lost at higher frequencies. Amplitude of oscillation was set to attain proper arterial $\mathrm{PCO}_{2}$ and was maintained between 40 and $55 \mathrm{mmHg}$. Immediately after intubation, MAP was set at $10 \mathrm{~cm} \mathrm{H}_{2} \mathrm{O}$ and increased stepwise by $1-2 \mathrm{~cm} \mathrm{H}_{2} \mathrm{O}$ per step until the $\mathrm{FIO}_{2}$ decreased below 0.30 (optimum lung volume strategy). Oxygen saturation was assessed using a pulse oximeter and was maintained between 90 and $95 \%$ (high lung volume strategy). Only at this point exogenous surfactant was administrated to the lungs through the endotracheal tube. The neonate remained at the supine position and was not tilted towards the side during this procedure. Thereafter, MAP was decreased in a stepwise fashion by $1-2 \mathrm{~cm} \mathrm{H}_{2} \mathrm{O}$ per step, as long as oxygenation did not deteriorate.

The acoustic sensor was attached on the chest near the right lung, $1 \mathrm{~cm}$ under the right nipple. The sensor was isolated from the subject's skin by two layers of 'Tegaderm' sterile tapes (3M Health Care). One 'Tegaderm' tape was attached directly to the neonate skin, and second tape covered the acoustic sensor, and used for attaching it to the chest. This method minimized the possibility of creating air holes between the transmitter and the fetal skin. A special hole was made in the second tape for allowing air passage between the transmitter and the ambient air. The attachment of the acoustic sensor was performed by the neonatologist, in a manner that did not apply external pressure on the chest.

In two of the cases, acoustic measurements started before the initiation of the recruitment maneuver and the administration of exogenous surfactant. In these cases the period of measurement included the standard process of the adjustment of ventilator parameters (including MAP), the administration of exogenous surfactant, and the process of surfactant distribution in the lungs. In the other four cases the acoustic measurements began immediately after the administration of surfactant. This was due to the fact that the procedure of attaching the acoustic sensor is time-consuming and interferes with the emergent procedure needed. In these cases the acoustic measurements were carried out during the final procedure of setting of MAP. In all cases, the session of acoustic transmission lasted more than 4 minutes. Between sessions, the acoustic sensor remained attached to the neonate chest, but the transmitter was off. In each case, one 
Table 1. Demographic Data and Measurements of Studied Infants

\begin{tabular}{|c|c|c|c|c|c|}
\hline Subject & $\begin{array}{c}\text { Gestational Age } \\
\text { (weeks) }\end{array}$ & Birth Weight (grams) & $\operatorname{MAP}$ Used $^{\mathrm{a}}\left(\mathrm{cmH}_{2} \mathrm{O}\right)$ & Calc. UIP ${ }^{\mathrm{b}}\left(\mathrm{cmH}_{2} \mathrm{O}\right)$ & Delta MAP ${ }^{\mathrm{c}}\left(\mathrm{cmH}_{2} \mathrm{O}\right)$ \\
\hline 1 & 31 & 1147 & 7.5 & 9.6 & 1.9 \\
\hline 2 & 22 & 600 & 12 & $>11$ & N/A \\
\hline 3 & 34 & 2334 & 11 & $>13$ & $>2$ \\
\hline 4 & 32 & 2000 & 9.7 & 11 & 1.3 \\
\hline 5 & 28 & 1340 & 9.5 & $>12$ & $>2.5$ \\
\hline 6 & 29 & 1169 & 7.8 & $>12$ & $>4.2$ \\
\hline
\end{tabular}

${ }^{a}$ The value of mean airway pressure determined by the neonatologist as the mean ventilation pressure

${ }^{\mathrm{b}}$ The value of UIP, as calculated by the normalized acoustic reflection-pressure curve

${ }^{c}$ Difference between MAP used and calculated UIP

minute of background noise in the NICU room was recorded. Standard medical treatment was not delayed or discontinued for the purpose of allowing measurement to be performed.

\subsection{Data Analysis}

The acoustic signals were transformed into the frequency domain using a fast Fourier transform algorithm, with frequency resolution of $39 \mathrm{~Hz}$. The power spectra were calculated using Welch's method and averaged over segments of $1 \mathrm{cmH}_{2} \mathrm{O}$ of MAP, deriving an average frequency spectrum for respective values of lung mean airway pressure. The amplitude of maximal intensity was used in each curve to assess sound reflection. For each subject, the value of the minimal MAP measured was determined. In this pressure, lung volume was expected to be minimal. The minimal MAP used, and its respective averaged acoustic spectrum were used as reference values for the 'empty lung' state.

To compare the results between subjects, we normalized the reflection-pressure relationship. This eliminated biases which may have resulted from intra-subject differenced in acoustic coupling between the acoustic sensor and the chest, ambient noise and differences in lung size between subjects. Sound reflection was set as 0 for the 'empty lung' state, and as 1 when the acoustic reflection was maximal. This procedure derived the reflection-pressure curves. In the normalized reflection curve, UIP was defined as the pressure in which the steepness of the reflection-pressure curve reduced to 0.15 of its maximal value.

\section{RESULTS}

The study group included six newborns who were admitted to the NICU at the Edith Wolfson Medical Center (Holon, Israel) and required mechanical ventilation. All subjects were in their first day of life, weighing between 600 and 2334 grams (mean weight of 1431 grams, with standard deviation of 630 grams) and were ventilated using HFOV. All subjects suffered from RDS and required high frequency oscillatory ventilation. Demographic data and MAP measurements are shown in Table 1. 'MAP used' refers to the value of MAP set by the neonatologist; 'Calc. UIP' refers to the value of the upper inflection point as calculated by the normalized acoustic reflection-pressure curve; 'Delta' is the difference between MAP used and calculated UIP. Subjects 4-6 was studies twice; before and after the administration of surfactant.

Fig. (3) shows changes in acoustic reflection during lung inflation in all subjects. Data is presented in the frequency domain. All measurements shown in this figure were carried out in the right lung, after the administration of exogenous surfactant. Baseline response in all subjects was calculated as the acoustic response in the lowest value of MAP used in the specific subject. Data is displayed on a three-dimensional grid, where reflection is a function of MAP and frequency.

Changes in acoustic reflection were of similar pattern in all subjects. An increase in reflection was observed in response to an increase in mean airway pressure. Maximal spectral density of the reflected signal in all levels of MAP was measured at the frequency of $2500 \mathrm{~Hz}$ (same as the transmitted signal). In subject \#1 acoustic reflection was measured also around $3500 \mathrm{~Hz}$. In relatively high levels of MAP (MAP> $10 \mathrm{cmH}_{2} \mathrm{O}$ ) the change in acoustic reflection became less significant and can be seen as a plateau in the three-dimensional curves.

Fig. (4) presents the normalized reflection-pressure curves of all subjects. These curves represent the relationship between the amplitude of acoustic reflection and pressure applied to lung (MAP). Each curve is normalized by its maximal amplitude of reflection, and presented between the range of 0 and 1 . It can be seen that all patterns are similar. This is expected since these two-dimensional curves are cross-section views of the 3D curves shown in Fig. (3). The acoustic reflection-pressure curves were of the same shape of the mechanical volume-pressure curve, with a linear region in the middle pressure range. In high values of MAP, the reflection-pressure curve extended into a plateau.

In two of the subjects acoustic measurements were carried out also before the administration of exogenous surfactant. Fig. (5) compares changes in acoustic reflection before and after the administration of surfactant. Top panel (a) presents the data of subject 4 , whereas the bottom panel (b) presents the data collected from subject 6 . In this figure, the 

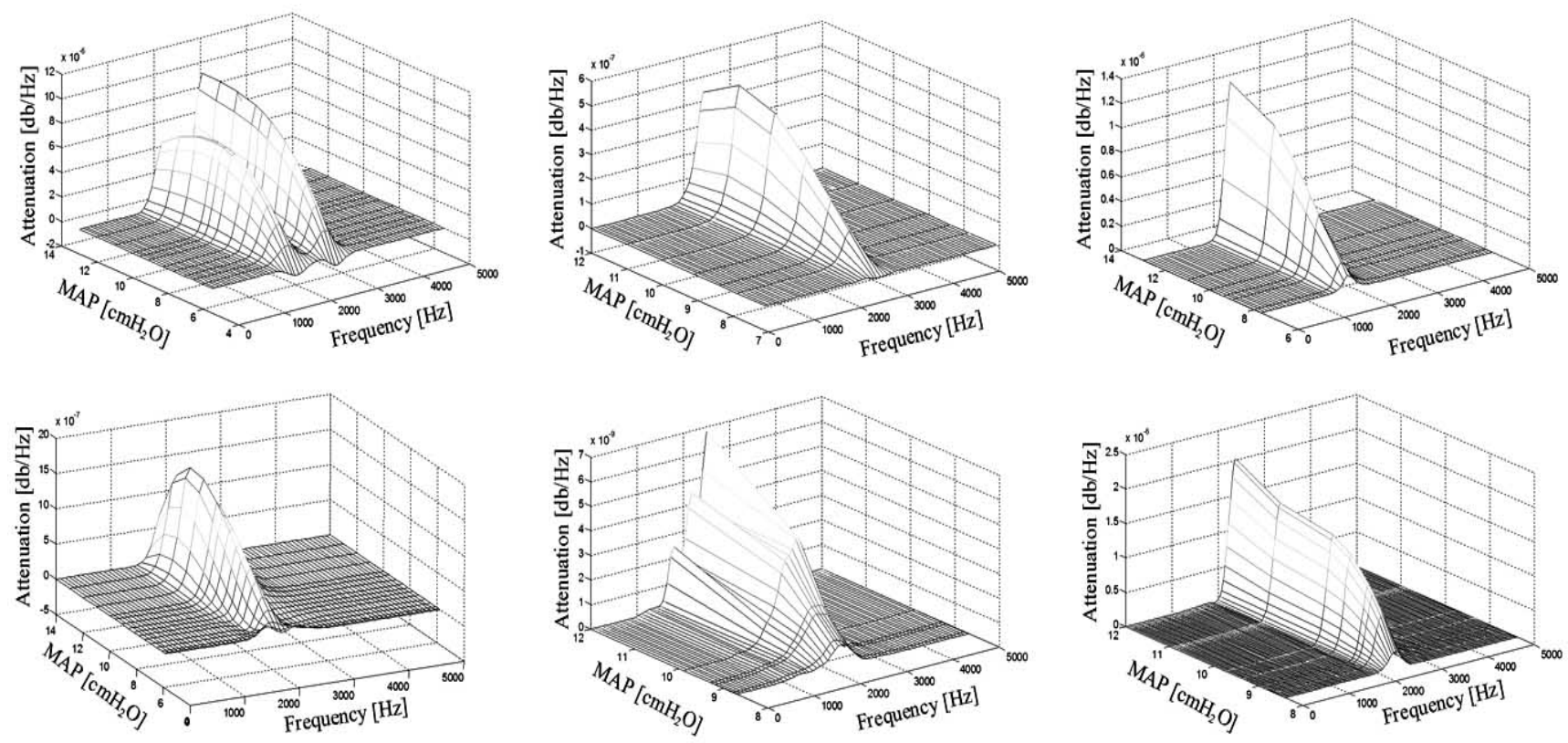

Fig. (3). Changes in acoustic reflection during lung inflation. Data is displayed on a three-dimensional grid, where reflection is function of MAP and frequency.

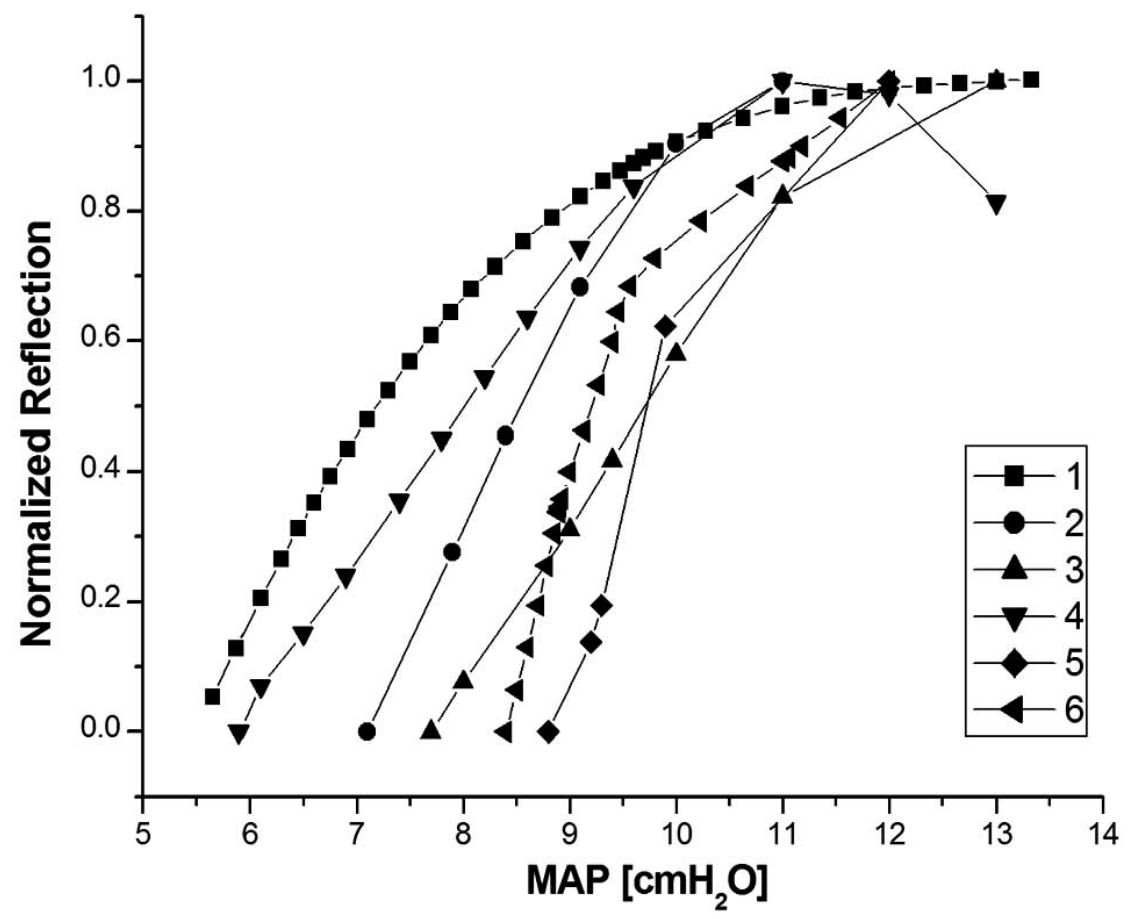

Fig. (4). Normalized reflection-pressure curves of all subjects, by their number.

data presented were the percentage of change in acoustic reflection (percent change). Baseline for all curves was defined as the amplitude of acoustic reflection of the 'empty' lung. As can be seen, in both subjects, maximal amplitude of lung reflection was higher after the administration of surfactant. In subject \#4 the change in acoustic reflection was more pronounced than in subject \#6 (Percent change of $250 \%$ compared to $17 \%$ ). This correlates with the differences in subjects' weight and gestation age (2000 grams, 32 weeks; compared to 1169 grams and 29 weeks, respectively).

Lung hysteresis in the volume-pressure plane was observed in two of the subjects when the lungs recruitment shifted from FRC to total lung capacity and then deflated. Fig. (6) shows how this cyclic change in lung pressure was expressed by the acoustic reflection, in subject 4. MAP was decreased from $10 \mathrm{cmH}_{2} \mathrm{O}$ to $5.5 \mathrm{cmH}_{2} \mathrm{O}$ (FRC), then in- 

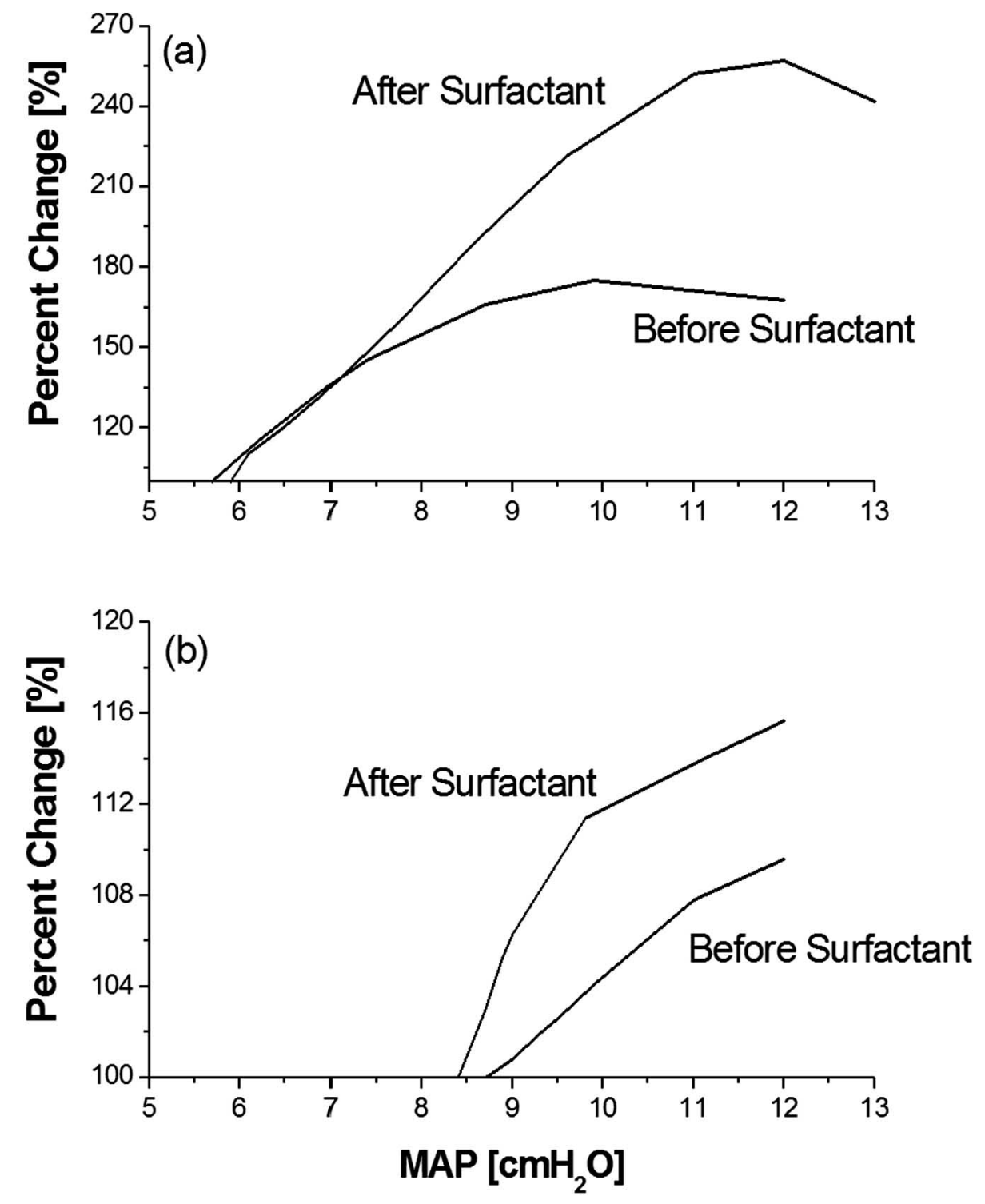

Fig. (5). Acoustic reflection before and after the administration of exogenous surfactant. Top panel (a) shows results of subject \#4, bottom panel (b) shows results of subject \#6.

creased to $14 \mathrm{cmH}_{2} \mathrm{O}$, and decreased again to $7 \mathrm{cmH}_{2} \mathrm{O}$. Sound reflection decreased with the decrease in MAP, and increased with the increase in MAP. However, level of sound reflection was higher along the deflation limb of the lung.

Calculated values of the upper inflection point in each subject were compared to the values of MAP chosen by the treating neonatologist during HFOV. Results are presented in Table 1. In all subjects (except for subject 2, where UIP was not reached), the value of MAP chosen by the neonatologist was lower than the calculated UIP. This indicated that the upper limit which was calculated according to sound reflection correlates with the physician's estimation of the mechanical UIP.

\section{DISCUSSION}

We hypothesized that sound reflection from the neonate lung can be used as an indirect index for estimation of lung volume during HFOV. Results of the feasibility experiments indicate that the acoustic properties of the newborn lungs indeed reflect the degree to which the lungs were inflated. Our study suggests that reflection of audible sound from the lung may be helpful in preterm RDS infants that are ventilated using HFOV and can be used to determine the optimal value of MAP that allows to maintain adequate lung volume and reduces the risk of either overdistension or underinflation.

The present study of the acoustic properties of the lung was motivated by a desire to find a method for monitoring lung volume of neonates ventilated by HFOV. The main finding that emerged from our study was that lung inflation resulted in a pronounced change in the acoustic properties of the sick neonatal lung. More specifically, we demonstrated that the reflection of acoustic signals increased with the increase in lung recruitment following changes in MAP. The relation between sound reflection and MAP, as shown in 

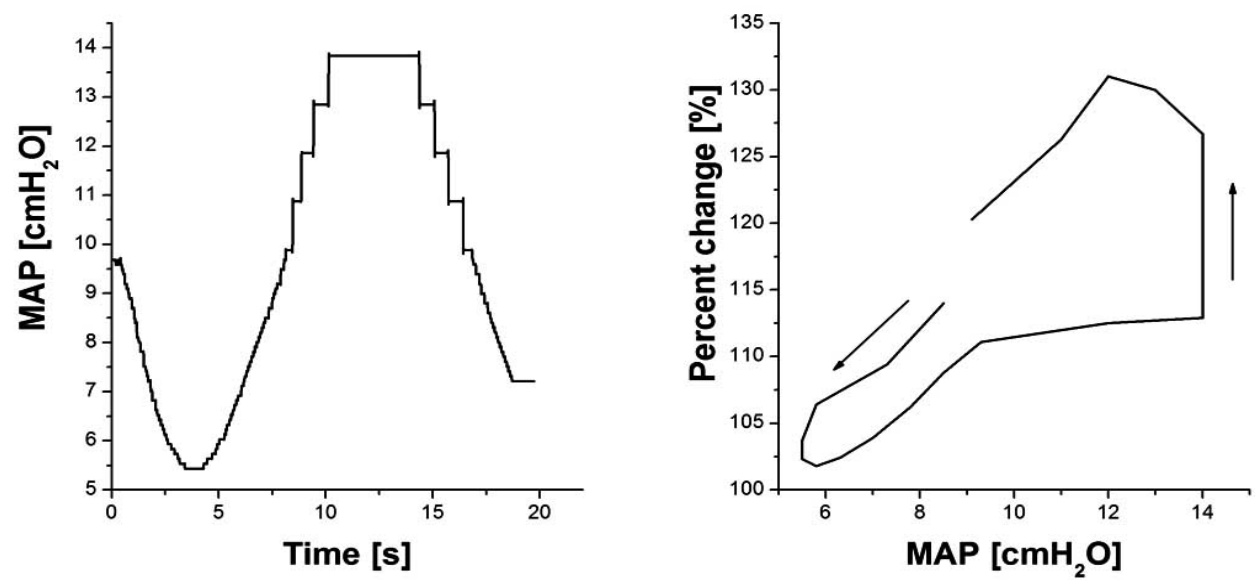

Fig. (6). Acoustic reflection during cyclic maneuver in MAP. Left panel: MAP, right panel: percent change in acoustic reflection.

Figs. (3 and 4) had a similar pattern to the known static volume-pressure curve. In the range of medium-to-high MAP, acoustic reflection increased linearly to the estimated change in lung volume. At high pressures, around the UIP, sound reflection reached a plateau, indicating that maximal lung recruitment was achieved. Sound reflection was not measured at pressures below the LIP since this pressure range is avoided in the clinical settings, as it may result in neonatal hypoxia.

We speculate that the change in sound reflection results from the increase in lung surface area. Below the LIP, the distal alveolar ducts are collapsed. When MAP increases, these ducts open and join the bronchial tree. This results in increased reflection of the acoustic wave from the surface of the lung. When the pressure reaches UIP, volume increase becomes small compared to the increase in pressure, and the change in sound reflection decreases.

Exogenous surfactant therapy has an established role in the management of RDS. It offers the advantage of establishing normal surfactant pools and improving lung mechanics. However, surfactant replacement therapy requires the placement of an endotracheal tube through which surfactant is directly instilled into the patient's lungs leading to alveolar stability. During the procedure, the dispersion of surfactant in the lung can not be estimated and the response to such therapy is established using clinical parameters such as improvement in oxygenation, a decrease in $\mathrm{FiO} 2$ and later a decrease in the value of MAP required for safe ventilation. Since the distribution of surfactant in the lungs results in an abrupt decrease in alveolar surface tension and increased lung volume [1], the rapid change in lung volume may be detected using acoustic measurements of the lung. The comparison of lung acoustics measurements before and after surfactant replacement therapy in our patients (Fig. 5) indicated a significant increase in lung volume and lung compliance following the procedure. Acoustic reflection was measured during epochs of a stepwise increase in MAP in all ventilated infants. MAP manipulation is associated with re-expansion of collapsed airways and alveoli and thus a pressure-volume relation of the lung is expected. Such a relationship was detected by our acoustic method (Fig. 6). This finding further supports the hypothesis that lung acoustics reflect lung volume.

When using analysis of sound reflection, one should be aware that the speed of audible sound transmission through the parenchyma is in the range of 2,300-7,000 cm/s [9]. Considering an average distance of $1-3 \mathrm{~cm}$ between the surface of the chest and the parenchymal-air interface, the minimal interval between transmission and reflection of the signal is less than 1 millisecond. Since the transmission burst interval is limited by the physical characteristics of the acoustic transmitter, the reflected signal may be received before the transmission is completed. In this type of measurement system, the recorded signal may consist two distinct components; a 'direct' transmitter-receiver component which results of a direct acoustic coupling between the transmitter and the receiver (direct propagation of sound from the transmitter to the receiver along the chest wall); and an 'indirect' (parenchymal-reflected) signal. While the first component is considered as constant and unaffected by changes in lung volume, the latter component reflects the anatomical changes of lung recruitment. One way to separate the parenchymalreflected component from the raw signal is to subtract the average signal of the lowest value of MAP (representing the acoustic properties of the 'empty' lung) from each average signals representing the acoustic properties of the lung during the process of lung recruitment.

Some limitations of the method presented here should be noted. Since sample size is small and heterogeneous, inference of results is limited. We assumed that the amplitude of sound reflection is correlative to the parenchymal density. The reflection-pressure relationships resembled the expected curves of volume-pressure. However, this could not be truly validated since the absolute volume of the lung could not be measured. From a technical aspect, there is an inherent limitation in resolution because the acoustic signal is of very low frequency. The technique can provide a reliable assessment of recruitment in the lung as a unified unit while local phenomena such as uneven expansion of lung units may not be detected. Improving the spatial resolution still poses a significant technical challenge. Also, since the acoustic measurements are taken in the very first minutes of ventilation simultaneously with other medical procedures, ambient noise 
and motion artifacts may affect the acoustic measurements. The rate in which MAP is changed during this process is a crucial factor for the signal to noise ratio (SNR) since it determines the amount of impulses averaged in each pressure segment.

Further clinical experiments are needed to establish objective criteria for determining the optimal value of MAP using the acoustic properties of the lung. Comparing the results of the acoustic technique with objective assessments of the lung volume such as CT is also necessary. Thus, we conclude that acoustic probing of the neonate lung undergoing HFOV has potential as an indicator of the degree of lung recruitment and may be used to define the optimal region of MAP in the neonatal sick lung.

\section{CONCLUSION}

The results of this feasibility experiment indicate that the acoustic properties of the newborn lungs reflect the degree to which the lungs are inflated. Our study suggests that reflection of audible sound from the lung may be helpful in preterm RDS infants that are ventilated using HFOV and can be used to determine the optimal value of MAP that allows to maintain adequate lung volume and reduces the risk of either overdistension or underinflation.

\section{REFERENCES}

[1] Dreyfuss D, Saumon G. Ventilator-induced lung injury: lessons from experimental studies. Am J Respir Crit Care Med 1998; 157(1): 294-323.

[2] Slutsky AS, Drazen JM. Ventilation with small tidal volumes. N Engl J Med. 2002 29; 347(9): 630-1.

[3] Pillow JJ. High-frequency oscillatory ventilation: mechanisms of gas exchange and lung mechanics. Crit Care Med 2005; 33(3 Suppl): S135-41.

[4] Courtney SE, Durand DJ, Asselin JM, Hudak ML, Aschner JL, Shoemaker CT. High-frequency oscillatory ventilation versus conventional mechanical ventilation for very-low-birth-weight infants. N Engl J Med 2002; 347(9): 643-52.

[5] Rimensberger PC, Beghetti M, Hanquinet S, Berner M. First intention high-frequency oscillation with early lung volume optimization improves pulmonary outcome in very low birth weight infants with respiratory distress syndrome. Pediatrics 2000; 105(6): 12028.

[6] Goddon S, Fujino Y, Hromi JM, Kacmarek RM. Optimal mean airway pressure during high-frequency oscillation: predicted by the pressure-volume curve. Anesthesiology 2001; 94(5): 862-9.

[7] Whitehead T, Slutsky AS. The pulmonary physician in critical care * 7: ventilator induced lung injury. Thorax 2002; 57(7): 635-42.

[8] Moloney ED, Griffiths MJ. Protective ventilation of patients with acute respiratory distress syndrome. Br J Anaesth 2004; 92(2): 26170.

[9] Amato MB, Barbas CS, Medeiros DM, et al. Beneficial effects of the "open lung approach" with low distending pressures in acute respiratory distress syndrome. A prospective randomized study on mechanical ventilation. Am J Respir Crit Care Med 1995; 152(6 Pt 1): $1835-46$.

[10] Bryan AC, Slutsky AS. Long volume during high frequency oscillation. Am Rev Respir Dis 1986; 133(5): 928-30.

[11] Froese AB. High-frequency oscillatory ventilation for adult respiratory distress syndrome: let's get it right this time! Crit Care Med 1997; 25(6): 906-8.

[12] Caironi P, Gattinoni L. How to monitor lung recruitment in patients with acute lung injury. Curr Opin Crit Care 2007; 13(3): 338-43.

[13] Thome U, Topfer A, Schaller P, Pohlandt F. Comparison of lung volume measurements by antero-posterior chest X-ray and the SF6 washout technique in mechanically ventilated infants. Pediatr Pulmonol 1998; 26(4): 265-72.

[14] Gothberg S, Parker TA, Griebel J, Abman SH, Kinsella JP. Lung volume recruitment in lambs during high-frequency oscillatory ventilation using respiratory inductive plethysmography. Pediatr Res 2001; 49(1): 38-44.

[15] Tingay DG, Mills JF, Morley CJ, Pellicano A, Dargaville PA. The deflation limb of the pressure-volume relationship in infants during high-frequency ventilation. Am J Respir Crit Care Med 2006; 173(4): 414-20.

[16] Dunlop S, Hough J, Riedel T, Fraser JF, Dunster K, Schibler A. Electrical impedance tomography in extremely prematurely born infants and during high frequency oscillatory ventilation analyzed in the frequency domain. Physiol Meas 2006; 27(11): 1151-65.

[17] Rice DA. Sound speed in pulmonary parenchyma. J Appl Physiol 1983; 54(1): 304-8.

[18] Wodicka GR, Stevens KN, Golub HL, Cravalho EG, Shannon DC. A model of acoustic transmission in the respiratory system. IEEE Trans Biomed Eng 1989; 36(9): 925-34.

[19] Wodicka GR, Aguirre A, DeFrain PD, Shannon DC. Phase delay of pulmonary acoustic transmission from trachea to chest wall. IEEE Trans Biomed Eng 1992; 39(10): 1053-9.

[20] Leung A, Sehati S, Young JD, McLeod C. Sound transmission between 50 and $600 \mathrm{~Hz}$ in excised pig lungs filled with air and helium. J Appl Physiol 2000; 89(6): 2472-82.

[21] Goncharoff V, Jacobs JE, Cugell DW. Wideband acoustic transmission of human lungs. Med Biol Eng Comput 1989; 27(5): $513-$ 9.

[22] Bergstresser T, Ofengeim D, Vyshedskiy A, Shane J, Murphy R. Sound transmission in the lung as a function of lung volume. $\mathbf{J}$ Appl Physiol 2002; 93(2): 667-74.

[23] Harper P, Kraman SS, Pasterkamp H, Wodicka GR. An acoustic model of the respiratory tract. IEEE Trans Biomed Eng 2001; 48(5): 543-50.

[24] Pasterkamp H, Powell RE, Sanchez I. Lung sound spectra at standardized air flow in normal infants, children, and adults. Am J Respir Crit Care Med 1996; 154(2 Pt 1): 424-30.

[25] Berger PJ, Skuza EM, Ramsden CA, Wilkinson MH. Velocity and attenuation of sound in the isolated fetal lung as it is expanded with air. J Appl Physiol 2005; 98(6): 2235-41.

[26] Rasanen J, Gavriely N. Detection of porcine oleic acid-induced acute lung injury using pulmonary acoustics. J Appl Physiol 2002; 93(1): 51-7.

[27] Royston TJ, Zhang X, Mansy HA, Sandler RH. Modeling sound transmission through the pulmonary system and chest with application to diagnosis of a collapsed lung. J Acoust Soc Am 2002; 111(4): 1931-46.

[28] Kompis M, Pasterkamp H, Wodicka GR. Acoustic imaging of the human chest. Chest 2001; 120(4): 1309-21.

(C) Luria et al.; Licensee Bentham Open.

This is an open access article licensed under the terms of the Creative Commons Attribution Non-Commercial License (http://creativecommons.org/licenses/by-nc/3.0/) which permits unrestricted, non-commercial use, distribution and reproduction in any medium, provided the work is properly cited. 